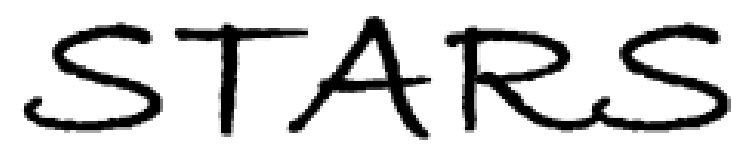

University of Central Florida

STARS

$1-1-2006$

\title{
Studies of minority carrier diffusion length increase in p-type ZnO
}

: $\mathrm{Sb}$

O. Lopatiuk-Tirpak

University of Central Florida

L. Chernyak

University of Central Florida

\section{F. X. Xiu}

J. L. Liu

S. Jang

See next page for additional authors

Find similar works at: https://stars.library.ucf.edu/facultybib2000

University of Central Florida Libraries http://library.ucf.edu

This Article is brought to you for free and open access by the Faculty Bibliography at STARS. It has been accepted for inclusion in Faculty Bibliography 2000s by an authorized administrator of STARS. For more information, please contact STARS@ucf.edu.

\section{Recommended Citation}

Lopatiuk-Tirpak, O.; Chernyak, L.; Xiu, F. X.; Liu, J. L.; Jang, S.; Ren, F.; Pearton, S. J.; Gartsman, K.; Feldman, Y.; Osinsky, A.; and Chow, P., "Studies of minority carrier diffusion length increase in p-type ZnO : Sb" (2006). Faculty Bibliography 2000s. 6376.

https://stars.library.ucf.edu/facultybib2000/6376

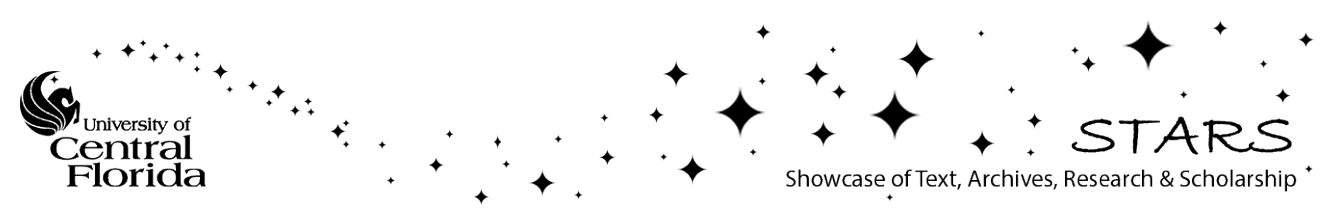


Authors

O. Lopatiuk-Tirpak, L. Chernyak, F. X. Xiu, J. L. Liu, S. Jang, F. Ren, S. J. Pearton, K. Gartsman, Y. Feldman, A. Osinsky, and P. Chow 


\section{Studies of minority carrier diffusion length increase in $p$-type ZnO:Sb}

Cite as: J. Appl. Phys. 100, 086101 (2006); https://doi.org/10.1063/1.2358844

Submitted: 19 July 2006 . Accepted: 14 August 2006 . Published Online: 23 October 2006

O. Lopatiuk-Tirpak, L. Chernyak, F. X. Xiu, J. L. Liu, S. Jang, F. Ren, S. J. Pearton, K. Gartsman, Y. Feldman, A. Osinsky, and P. Chow

\section{ARTICLES YOU MAY BE INTERESTED IN}

Diameter dependence of the minority carrier diffusion length in individual ZnO nanowires Applied Physics Letters 96, 253115 (2010); https://doi.org/10.1063/1.3456390

A comprehensive review of $\mathrm{ZnO}$ materials and devices

Journal of Applied Physics 98, 041301 (2005); https://doi.org/10.1063/1.1992666

Electron-beam-induced current and cathodoluminescence studies of thermally activated increase for carrier diffusion length and lifetime in $n$-type $\mathrm{ZnO}$

Applied Physics Letters 87, 162103 (2005); https://doi.org/10.1063/1.2106001

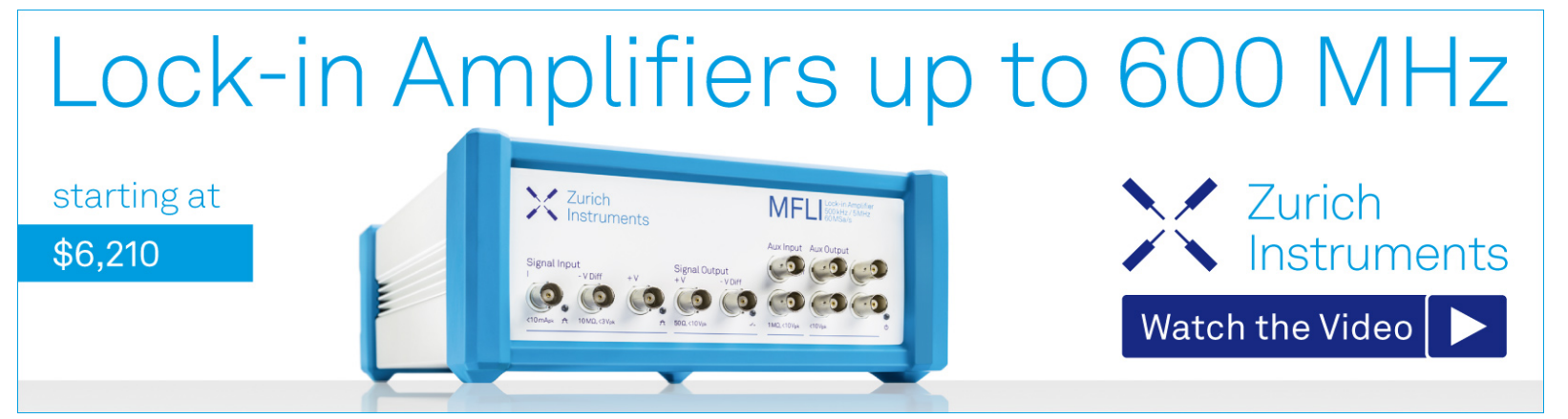

J. Appl. Phys. 100, 086101 (2006); https://doi.org/10.1063/1.2358844

100,086101

(c) 2006 American Institute of Physics. 


\title{
Studies of minority carrier diffusion length increase in p-type $\mathrm{ZnO}: \mathrm{Sb}$
}

\author{
O. Lopatiuk-Tirpak and L. Chernyak ${ }^{\text {a) }}$ \\ Department of Physics, University of Central Florida, Orlando, Florida 32816-2385 \\ F. X. Xiu and J. L. Liu \\ Department of Electrical Engineering, University of California, Riverside, California 92521 \\ S. Jang, F. Ren, and S. J. Pearton \\ Department of Materials Science and Engineering, University of Florida, Gainesville, Florida 32611 \\ K. Gartsman and Y. Feldman \\ Weizmann Institute of Science, Rehovot 76100, Israel
}

A. Osinsky and P. Chow

SVT Associates, Eden Prairie, Minnesota 55344

(Received 19 July 2006; accepted 14 August 2006; published online 20 October 2006)

\begin{abstract}
Minority electron diffusion length was measured in $p$-type, Sb-doped $\mathrm{ZnO}$ as a function of temperature using the electron beam induced current technique. A thermally induced increase of electron diffusion length was determined to have an activation energy of $184 \pm 10 \mathrm{meV}$. Irradiation with a low energy $(5 \mathrm{kV})$ electron beam also resulted in an increase of diffusion length with a similar activation energy $(219 \pm 8 \mathrm{meV})$. Both phenomena are suggested to involve a $\mathrm{Sb}_{\mathrm{Zn}}-2 \mathrm{~V}_{\mathrm{Zn}}$ acceptor complex. Saturation and relaxation dynamics of minority carrier diffusion length are explored. Details of a possible mechanism for diffusion length increase are presented. (C) 2006 American Institute of Physics. [DOI: 10.1063/1.2358844]
\end{abstract}

The realization of the full potential of $\mathrm{ZnO}$ in bipolar device applications has long been challenged by the availability of high-quality, highly conductive $p$-type material. Several studies have shown that doping with group V impurities (phosphorus ${ }^{1-3}$ arsenic, $^{4,5}$ and antimony ${ }^{6,7}$ ) can yield materials with reasonably high hole concentrations, despite the large mismatch in ionic radii. First-principles investigation into $p$-type conductivity observed experimentally in $\mathrm{ZnO}: \mathrm{As}$ and $\mathrm{ZnO}: \mathrm{Sb}$ suggested that the nature of acceptors in these materials may be different from the simple substitution on oxygen site; namely, it was found that an acceptor complex of the impurity with two zinc vacancies $\left(X_{\mathrm{Zn}}-2 \mathrm{~V}_{\mathrm{Zn}}\right.$, where $X$ denotes As or Sb) has a low formation energy as well as a low ionization energy, which is consistent with the experimental observations. ${ }^{8}$

Although the feasibility of viable $\mathrm{ZnO}$-based homojunction devices is continually increasing due to advances in $p$-type doping, their performance may be fundamentally limited by the transport properties of minority carriers. In direct band gap semiconductors, including $\mathrm{ZnO}$, minority carrier diffusion length is generally several orders of magnitude lower than in indirect gap materials such as silicon or germanium.

Earlier cathodoluminescence studies performed on p-type $\mathrm{ZnMgO}: \mathrm{P}$ (Ref. 9) and bulk $\mathrm{ZnO}$ containing lithium ${ }^{10}$ revealed that lifetime of nonequilibrium carriers can be significantly extended by exposure to the beam of the scanning electron microscope (SEM). This phenomenon was attributed to the trapping of nonequilibrium electrons on deep, nonionized acceptor levels. In this work, we use a different

${ }^{a)}$ Electronic mail: chernyak@ physics.ucf.edu technique [electron beam induced current (EBIC)] to demonstrate that electron irradiation of $p$-type $\mathrm{ZnO}: \mathrm{Sb}$ leads to a marked increase in the diffusion length of minority electrons and provide further evidence of the role of deep acceptor levels in irradiation-induced behavior by performing temperature-dependent measurements.

The experiments were performed on $0.2-\mu \mathrm{m}$-thick $\mathrm{ZnO}: \mathrm{Sb}$ thin film grown on $\mathrm{Si}$ (100) substrate by an electron cyclotron resonance (ECR)-assisted molecular beam epitaxy (MBE). The detailed growth procedures are available in Ref. 6. Hall effect measurements revealed strong $p$-type conductivity, with hole concentration of $1.3 \times 10^{17} \mathrm{~cm}^{-3}$ and mobility of $28.0 \mathrm{~cm}^{2} / \mathrm{V} \mathrm{s}$ at room temperature.

Minority carrier diffusion length $L$ was determined using EBIC line-scan method. ${ }^{11}$ Schottky contacts for EBIC measurements were prepared by lift-off of $e$-beam evaporated $\mathrm{Pt} / \mathrm{Au}(500 \AA / 1000 \AA)$. All experiments were carried out in situ in a Philips XL30 SEM using accelerating voltage of $5 \mathrm{kV}$, which corresponds to the electron range of $\sim 0.15 \mu \mathrm{m}$ in $\mathrm{ZnO}$ epitaxial layer. After initial line-scan (24 s) EBIC measurement and extraction of $L$, the electron beam irradiation was continued by repeating the line scan over the same location for about $2000 \mathrm{~s}$, with additional EBIC measurements performed intermittently. The sample temperature was varied from 0 to $100{ }^{\circ} \mathrm{C}$ using an external temperature controller (Gatan), with a new area for electron beam irradiation chosen at each temperature.

Before investigating the dependence of $L$ on electron beam irradiation, the impact of sample temperature $T$ on minority carrier diffusion length in $\mathrm{ZnO}: \mathrm{Sb}$ was determined. As can be seen from Fig. 1, the value of $L$ increases exponentially as the temperature is raised. This behavior is common 


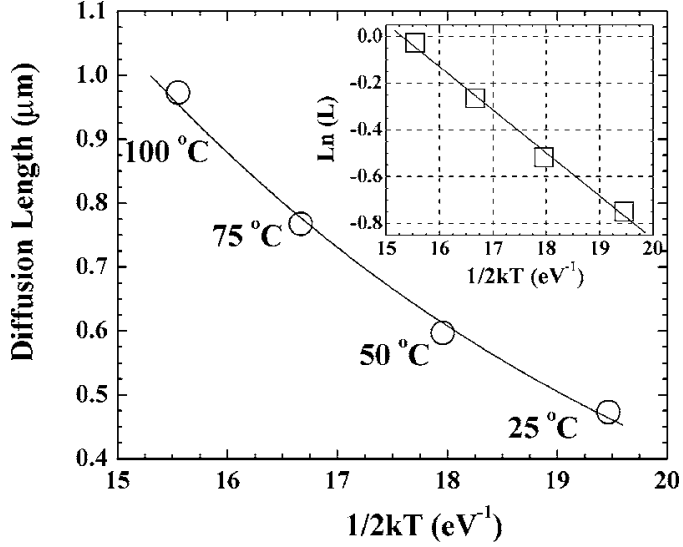

FIG. 1. Diffusion length of minority electrons as a function of temperature (open circles) and the fit with Eq. (1) (solid line). Inset: Arrhenius plot of the same data yielding activation energy of $184 \pm 10 \mathrm{meV}$.

to many semiconductors ${ }^{11,12}$ and is modeled by the following relationship:

$$
L=L_{0} \exp \left(-\frac{\Delta E_{A, T}}{2 k T}\right)
$$

where $L_{0}$ is a scaling factor, $k$ is Boltzmann constant, and $\Delta E_{A, T}$ is thermal activation energy. The latter parameter likely represents carrier delocalization energy and determines the increase of the diffusion length due to the reduction in recombination efficiency. ${ }^{12}$ Earlier photo- and cathodoluminescence studies on the similar samples had shown that the recombination route of nonequilibrium carriers involves transitions to a deep, neutral acceptor level $\left(e, \mathrm{~A}^{0}\right){ }^{6,13} \mathrm{An}$ increase in the temperature of the sample leads to a higher ionization fraction of the acceptors, reducing the concentration of $A^{0}$ and thus inhibiting the recombination rate. Lower recombination rate translates directly to greater lifetime of minority electrons in the conduction band (cf. Ref. 13) and, consequently, to greater diffusion length, since $L=(D \tau)^{1 / 2}$, with $D$ being the diffusivity of the carriers. From the Arrhenius plot shown in the inset of Fig. 1, the value of $\Delta E_{A, T}$ was determined to be $184 \pm 10 \mathrm{meV}$ and is in reasonable agreement with the activation energy of temperature-induced lifetime increase obtained in Ref. 13.

Continuous irradiation of the sample by the electron beam of the SEM was also shown to result in an increase of the minority carrier diffusion length. The irradiation was performed at several temperatures, with the diffusion length exhibiting a linear trend, as shown in Fig. 2. Note that the values of $L$ were vertically offset to clearly show its increase with duration of irradiation and thus do not accurately represent the temperature dependence of diffusion length.

The irradiation-induced increase of $L$ is thought to have an origin similar to that of the thermally induced increase: the nonequilibrium electrons generated by the electron beam are continuously trapped by the neutral acceptor levels $\left(A^{0}\right.$ $\left.+e^{-} \rightarrow A^{-}\right)$, reducing the recombination rate and resulting in longer lifetime of electrons in the conduction band. As excitation proceeds, the concentration of neutral levels diminishes and the increase of $L$ continues.

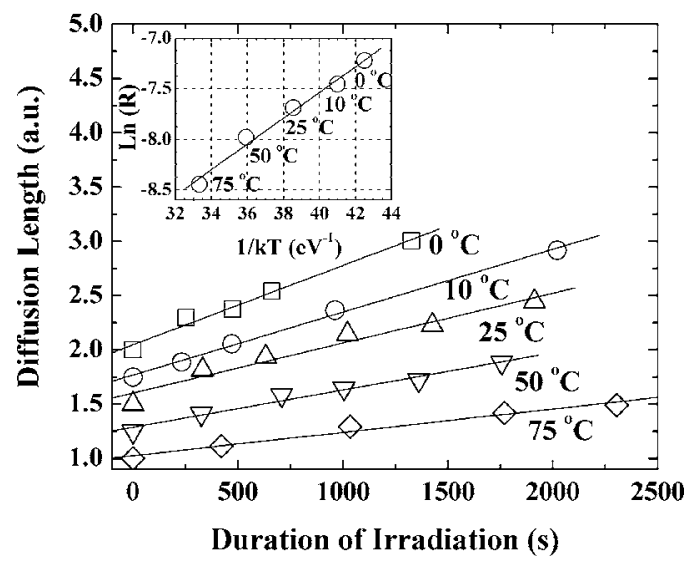

FIG. 2. Electron irradiation-induced increase of minority electron diffusion length at different temperatures. The values of the diffusion length were vertically offset for clarity and are not intended to illustrate the temperature dependence. Inset: Rate of irradiation-induced increase of diffusion length as a function of temperature (open circles). The fit with Eq. (2) (solid line) gives an activation energy of $219 \pm 8 \mathrm{meV}$.

It can also be seen from Fig. 2 that the rate $R$ of the diffusion length increase is reduced with increasing temperature. The increase of the diffusion length due to trapping is counteracted by the release of the trapped electrons that occurs if the carriers gain sufficient energy to escape the trap. As the temperature is raised, the likelihood of detrapping increases, which dampens the irradiation-induced growth of the diffusion length.

The influence of electron irradiation and temperature on the rate $R$ can be summarized by the following expression: ${ }^{12}$

$$
R=R_{0} \exp \left(\frac{\Delta E_{A, I}}{k T}\right) \exp \left(-\frac{\Delta E_{A, T}}{2 k T}\right),
$$

where $R_{0}$ is a scaling constant, $\Delta E_{A, I}$ is the activation energy of electron irradiation effect, and $\Delta E_{A, T}$ is the thermal activation energy determined as described above. Using the above equation, the activation energy of irradiation-induced component of diffusion length increase can be obtained from the Arrhenius plot shown in the inset of Fig. 2. The value of $\Delta E_{A, I}$ can be extracted from the slope of the linear fit, which from Eq. (2) equals $\Delta E_{A, I}-(1 / 2) \Delta E_{A, T}$. The treatment yielded a value of $219 \pm 8 \mathrm{meV}$, which is similar to the thermal activation energy obtained above and is in excellent agreement with that obtained in Ref. 13 (212 $28 \mathrm{meV})$ from cathodoluminescence measurements, which further supports the involvement of the same acceptor level in both irradiation- and temperature-induced processes.

The saturation and relaxation of irradiation-induced change of diffusion length was also studied. Figure 3 demonstrates that $L$ reaches its maximum value after about $50 \mathrm{~min}$ of continuous exposure to the electron beam. Further monitoring revealed that irradiation-induced increase persists for at least one week. Annealing the sample at $175^{\circ} \mathrm{C}$ for about $30 \mathrm{~min}$ resulted in a decrease of the room-temperature diffusion length to about $1 \mu \mathrm{m}$. This behavior further supports the involvement of deep electron traps in the phenomenon of interest, since temperature-induced detrapping of carriers reactivates the original recombination route, thus reducing carrier lifetime and diffusion length. 


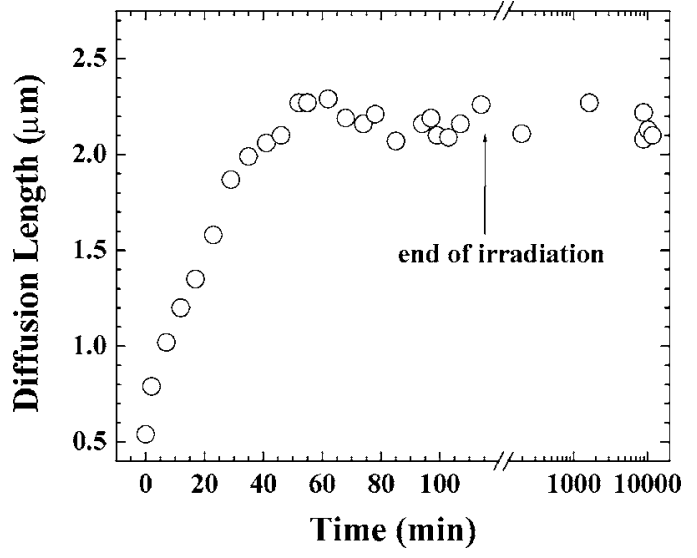

FIG. 3. Saturation and relaxation dynamics of minority carrier diffusion length. The arrow marks the time at which the electron irradiation was discontinued.

While the nature of the acceptor responsible for the increase of $L$ cannot be deduced with absolute certainty, experimental evidence obtained in this and other studies ${ }^{6,13}$ suggests that it is a $\mathrm{Sb}_{\mathrm{Zn}}-2 \mathrm{~V}_{\mathrm{Zn}}$ complex, predicted by Limpijumnong $e t a l{ }^{8}$ to have the activation energy of about $160 \mathrm{meV}$. As this value is somewhat lower than that determined by our experiments, it should be noted that it does not account for the strong dependence of the acceptor activation energy on the concentration of majority carriers. It has been shown that in $\mathrm{Sb}$-doped $\mathrm{ZnO}$, acceptor activation energy can increase from about 135 to $212 \mathrm{meV}$ in the range of carrier concentrations spanning about one order of magnitude. ${ }^{13}$ Therefore, the above theoretical prediction does not necessarily contradict the experimental results. On the other hand, the involvement of other Sb-related defects is highly unlikely. The substitutional defect $\left(\mathrm{Sb}_{\mathrm{O}}\right)$ as well as the singlevacancy complex $\left(\mathrm{Sb}_{\mathrm{Zn}}-\mathrm{V}_{\mathrm{Zn}}\right)$ are predicted to have ionization energies about an order of magnitude greater than those obtained experimentally, while other defects can also be ruled out based on their electrical behavior and/or high formation energies. ${ }^{8}$
In summary, temperature-dependent EBIC measurements allow us to estimate the activation energies of the thermally and electron irradiation-induced increase of the minority carrier diffusion length in $p$-type, Sb-doped $\mathrm{ZnO}$. Based on the values of the activation energy, both phenomena are suggested to occur due to carrier trapping on the deep $\mathrm{Sb}$-related acceptor level, which is likely a $\mathrm{Sb}_{\mathrm{Zn}}-2 \mathrm{~V}_{\mathrm{Zn}}$ complex.

The work at the University of Central Florida is supported in part by the National Science Foundation (ECS 0422604), the American Chemical Society Petroleum Research Fund (40501-AC10), and NATO (PST.MD.CLG 980579). The work at UC Riverside was supported by DARPA/DMEA through the center for NanoScience and Innovation for Defense (H94003-04-2-0404). The work at UF is partially supported by AFOSR grant under Grant No. F49620-03-1-0370, by the Army Research Office under Grant No. DAAD19-01-1-0603, NSF CTS-0301178 and the NSF DMR 0400416.

${ }^{1}$ F. G. Chen, Z. Z. Ye, W. Z. Xu, B. H. Zhao, L. P. Zhu, and J. G. Lv, J. Cryst. Growth 281, 458 (2005).

${ }^{2}$ Y. W. Heo, K. Ip, S. J. Pearton, and D. P. Norton, Phys. Status Solidi A 201, 1500 (2004).

${ }^{3}$ V. Vaithianathan, B. T. Lee, and S. S. Kim, J. Appl. Phys. 98, 043519 (2005).

${ }^{4}$ V. Vaithianathan, B. T. Lee, and S. S. Kim, Appl. Phys. Lett. 86, 062101 (2005).

${ }^{5}$ Y. R. Ryu, S. Zhu, D. C. Look, J. M. Wrobel, H. M. Jeong, and H. W. White, J. Cryst. Growth 216, 330 (2000).

${ }^{6}$ F. X. Xiu, Z. Yang, L. J. Mandalapu, D. T. Zhao, J. L. Liu, and W. P. Beyermann, Appl. Phys. Lett. 87, 152101 (2005)

${ }^{7}$ T. Aoki, Y. Shimizu, A. Miyake, A. Nakamura, Y. Nakanishi, and Y. Hatanaka, Phys. Status Solidi B 229, 911 (2002).

${ }^{8}$ S. Limpijumnong, S. B. Zhang, S. H. Wei, and C. H. Park, Phys. Rev. Lett. 92, 155504 (2004).

${ }^{9}$ O. Lopatiuk et al., Appl. Phys. Lett. 86, 012105 (2005).

${ }^{10}$ O. Lopatiuk, L. Chernyak, A. Osinsky, and J. Q. Xie, Appl. Phys. Lett. 87, 214110 (2005).

${ }^{11}$ L. Chernyak, A. Osinsky, H. Temkin, J. W. Yang, Q. Chen, and M. A. Khan, Appl. Phys. Lett. 69, 2531 (1996).

${ }^{12}$ M. Eckstein and H. U. Habermeier, J. Phys. IV 1, 23 (1991).

${ }^{13}$ O. Lopatiuk-Tirpak et al., Appl. Phys. Lett. 88, 202110 (2006). 